\title{
Sentinel Lymph Node Detection in Patients with Early Cervical Cancer
}

\author{
Acharya $B C,{ }^{1}$ Jihong $L^{2}$ \\ 'Department of Surgical Oncology, Gynae. Oncology Unit, B.P.Koirala Memorial Cancer Hospital, Bharatpur, Chitwan, \\ Nepal, ${ }^{2}$ Gynaecology Oncology Department, Sun Yat-sen University Cancer centre China.
}

\section{ABSTRACT}

Introduction: Lymph node status is the most important independent prognostic factor in early stage cervical cancer. Intraoperative lymphatic mapping and sentinel lymph node detection have been increasingly evaluated in the treatment of a variety of solid tumors, particularly breast cancer and cutaneous melanoma. This study evaluated the feasibility of these procedures in patients undergoing radical hysterectomy with pelvic lymphadenectomy for early cervical cancer.

Methods: A total of 30 patients with histologically diagnosed FIGO stage IA to IIA cervical cancer were enrolled to this study. They were scheduled to undergo radical abdominal hysterectomy and pelvic lymphadenectomy after injecting patent blue dye in cervix.

Results: A total of 60 SLNs (mean 2.5) were detected in 24 patients with detection rate of $80 \%$. Bilateral SLNs were detected in $70.1 \%$ of cases. SLNs were identified in obturator and external iliac areas in $50 \%$ and $31.7 \%$, respectively; no SLNs were discovered in the common iliac region. Seven patients (23.3\%) had lymph node metastases; one of these had false negative SLN.The false negative rate and negative predictive value were $14.3 \%$ and $94.4 \%$, respectively.

Conclusions: SLN detection procedure with blue dye technique is a feasible procedure in cervical cancer. Patent blue dye is cheap, safe and effective tracer to detect sentinel node in carcinoma of cervix.

Key Words: cervical cancer, lymphatic mapping, sentinel lymph node

\section{INTRODUCTION}

The sentinel lymph node (SLN) is the first node to receive lymphatic drainage from primary tumor, so that when nodal metastases occur, it will be first to be involved. In areas of lymphatic drainage (basins) where the SLN concept is valid, pathologic status of these specific nodes should reflect the overall status of the entire regional draining area. ${ }^{1}$
Cervical cancer is a good candidate disease for lymphatic mapping because of the following important reasons: firstly, because the incidence of nodal metastases is 0 $16 \%$ in patients with cervical tumor size less than or equal to $2 \mathrm{~cm}$ and $15-31 \%$ in patients with overall stage $\mathrm{IB}$; thus many patients with negative nodes will derive
Correspondence:
Dr. Bijaya Chandra Acharya
Department of Surgical Oncology
Gynaecology Oncology Unit
BP Koirala Memorial Cancer Hospital
Bharatpur, Chitwan, Nepal.
Email: bijacharya@hotamil.com
Phone: 9845024073 
no benefit, but will develop short and long-term morbidity related to Iymphadenectomy. Secondly, cervix has a complex lymphatic drainage due to its midline position. ${ }^{2}$ Thirdly; conventional imaging techniques notoriously fail to identify with accuracy lymph node metastases. The methods used in different studies were blue dye, radio colloid tracer and or a combination of both with using gamma probe detector. ${ }^{3}$

\section{METHODS}

This is a prospective study to detect SLN in early cervical cancer. After taking permission from hospital authority this study was performed at B.P.Koirala Memorial Cancer Hospital, Bharatpur from August 2005 to April 2006. Ethical approval and patient consent were taken. Patients with histologically proven FIGO stage IA to IIA, primary cervical cancer were eligible, if they were scheduled to undergo radical hysterectomy and systematic pelvic lymphadenectomy. Patients were considered ineligible if they had prior chemotherapy, pelvic radiotherapy or retroperitoneal surgery for possible alterations of pathological nodal status and lymphatic flow. All histological tumor types were included.

At the time of surgery, patients were placed in the low lithotomy position and under direct visualization, $4 \mathrm{ml}$ of patent blue dye $(1 \%)$ was injected into the cervix (depth of injection was 5-10 mm) using a 25-gauge standard sterile needle at 3, 6, 9, 0 o'clock positions of the cervix. Whenever possible, direct tumoral injection was avoided. Dye was injected slowly and with constant gentle pressure on the syringe to let the pick up of dye by the lymphatics. The abdomen was immediately opened by midline infraumbilical incision. The pelvic peritoneum of the right side was first opened and the avascular para-vesicle and para-rectal spaces were gently developed using blunt dissection. Blue lymphatics were followed all the way to their ending in a blue node; the lymph node with obvious or faint dye uptake, classified as SLN, was dissected and removed separately. The pelvic nodal "basin" was defined as all lymph node -bearing tissue in the internal and external iliac, obturator, and distal common iliac nodal chains. Before proceeding with systematic lymphadenectomy on the right side, the same steps were performed contra-laterally to avoid losing SLN on the left side. After the SLN procedure, systematic bilateral pelvic lymphadenectomy was done to remove all fatty lymphatic tissue in the area from the origin of common iliac artery to the circumflex iliac vein. The SLN procedure was considered as failed if no sentinel node was identified on either side of pelvic dissection. A "false negative" study was defined as one in which all sentinel nodes identified in a basin are histologically negative, but tumor is present in the other lymph node(s) from that same basin. Sentinel nodes were submitted for examination as separate specimen and remaining lymph nodes were sent separately with proper labeling.

\section{RESULTS}

The age, FIGO stage, histology type and TNM classifications depicts various pictures (Table 1). Mean age of the patients was 48.13 years and the range 32 67 years. A total of 553 pelvic lymph nodes (mean 18.43, range 9-38) were dissected in 30 patients. Non-SLNs totaled 493 (mean 16.43, range 8-37). A total of 60 SLNs (mean 2.5 per patient, range 1-6) were identified in 24 patients, whereas in 6 patients (20\% failure rate) no SLNs were detected. Overall detection rate was $80 \%$. Among the 24 patients, bilateral detection of SLN was in 17 patients (70.9\%). The most common site of SLNs was obturator and the external iliac areas $150 \%$ and $31.7 \%$, respectively). Remaining SLNs were detected in parametrial and internal iliac region (Table 2).

Table 1. Patient characteristic

\begin{tabular}{|c|c|c|c|}
\hline Case & Age (years) & FIGO stage & Histology type \\
\hline 1 & 36 & IA & SCC \\
\hline 2 & 40 & IIA & SCC \\
\hline 3 & 48 & IIA & AS \\
\hline 4 & 41 & IIA & SCC \\
\hline 5 & 50 & IB2 & SCC \\
\hline 6 & 50 & IB1 & SCC \\
\hline 7 & 32 & IB2 & SCC \\
\hline 8 & 50 & IB1 & ADENO \\
\hline 9 & 60 & IB1 & SCC \\
\hline 10 & 47 & IB1 & SCC \\
\hline 11 & 45 & IIA & SCC \\
\hline 12 & 45 & IB2 & SCC \\
\hline 13 & 45 & IIA & SCC \\
\hline 14 & 64 & IIA & SCC \\
\hline 15 & 45 & $\| \mathrm{A}$ & $\mathrm{SCC}$ \\
\hline 16 & 57 & IB1 & ADENO \\
\hline 17 & 51 & IIA & SCC \\
\hline 18 & 59 & IB1 & SCC \\
\hline 19 & 38 & IB1 & SCC \\
\hline 20 & 54 & IB1 & SCC \\
\hline 21 & 48 & IB2 & SCC \\
\hline 22 & 34 & IB1 & SCC \\
\hline 23 & 43 & IB1 & SCC \\
\hline 24 & 52 & IIA & SCC \\
\hline 25 & 55 & IIA & AS \\
\hline 26 & 54 & IB1 & SCC \\
\hline 27 & 45 & IB1 & AS \\
\hline 28 & 34 & $\| \mathrm{A}$ & SCC \\
\hline 29 & 67 & IIA & SCC \\
\hline 30 & 55 & IB2 & SCC \\
\hline
\end{tabular}

$S C C=$ squamous cell carcinoma; $A S=$ adenosquamous cell carcinoma; $A D E N O=$ adenocarcinoma.

\section{JNMA | Val 48}


Table 2. Site wise distribution of SLN

\begin{tabular}{lll}
\hline Site & No. of SLN & Percentage \\
\hline Obturator & 30 & 50 \\
External iliac & 19 & 31.7 \\
Parametrial & 6 & 10 \\
Internal iliac & 5 & 8.3 \\
Total & 60 & 100 \\
\hline
\end{tabular}

There were no differences in the distribution of lymphatic drainage area between right and left side of pelvis. It was considered "typical caudal nodes" those identified below the external iliac vein and those localized between external and internal iliac vessels (i.e.obturator and internal iliac groups), this group is also called "sub venous group". All these SLNs lie in the so-called "Leveuf and Godard" area, i.e., lateral to the inferior vesicle artery, ventral to the origin of the uterine artery, and medial or caudal to the external iliac vein. One of the SLNs was identified in the common iliac region. No dye uptake was seen in lymphatics draining towards the presacral or the lower aortic region. Blue lymph nodes were usually identified in the pelvis not less than 18 minutes and not more than 90 minutes after dye injection.

Seven patients $(23.3 \%)$ were found to have lymph node metastases. All node positive patients had lymphovascular space involvement. Among total 17 positive nodes, $15(88.29 \%)$ were sentinel nodes and remaining 2 (11.8\%) nodes were not sentinel nodes (Table 3 ).

Table 3. Staging of lymph node positive patients.

\begin{tabular}{lll}
\hline FIGO stage & No. of patients & Percentage \\
\hline IB1 & 3 & 42.8 \\
IB2 & 1 & 14.2 \\
IIA & 3 & 42.8 \\
Total & 7 & 100 \\
\hline
\end{tabular}

With one false-negative result in this series, the falsenegative rate and negative predictive value were $14.3 \%$ and $94.4 \%$ respectively. Accuracy of this study was $95.8 \%$.

\section{DISSCUSSION}

SLN detection with lymphatic mapping is a promising strategy for intraoperative assessment of nodal status in a variety of tumor types. SLNs detection technique has been applied successfully in treating breast cancers and cutaneous melanoma patients and may decrease morbidity by allowing omission of complete lymphadenectomy in some patients. ${ }^{4,5}$ Successful implementation of these techniques is dependent on operator skill as well as tumor specific factors, such as tumor size, predictability of the regional drainage pattern and accessibility of lymphatic basins. This study explored the feasibility of lymphatic mapping in patients undergoing radical hysterectomy and pelvic lymphadenectomy. If validated, a reliable lymphatic mapping strategy could alter the way certain patients with early cervical cancer are managed. Anatomists have established that lymph node drainage is predictable yet not uniform so that identification of the SLN as the indicator of a cervical metastases disease would be useful in order to improve the detection of cancer cells, spread and to reduce morbidity and mortality related to the procedure of its detection.

The term sentinel lymph node was coined in 1960 by Gould et.al.Validity of sentinel node concept was established subsequently in patients with melanoma and breast carcinoma and its feasibility are under evaluation in vulvar malignancies. ${ }^{6,7}$

In cervical cancer, lymphatic mapping is a promising strategy for intraoperative assessment of nodal status. Except for the first lymphatic mapping report in which the detection rate was only $15 \%$, the SLN detection rate range between $55.5 \%$ and $100 \%$, regardless of the surgical approach on the detection method. ${ }^{8}$ In this study, the SLN detection rate was $80 \%$.

In cervical cancer, at least one SLN should be found per side of dissection, because one cannot assume the SLN status on one side reflects the status on the other. In this series, unilateral SLN detection occurred in $29.19 \%$. This result could be explained by different studies ${ }^{9}$ performed by different authors.

Explanation for failure of detection of SLN could be inappropriate timing of reaching paraspaces. Like Dargent et al. $^{10}$ we noted that a window of 18 minutes was necessary to allow the passage of dye through cervical lympahatics into the parametrial tissues, and ultimately to the pelvic sidewall. However, we never detected any SLN after more than 90 minutes since the injection, which is a disagreement with Dargent, et al (150 minutes); this might be attributed to the laparotomic approach. It is possible that positive intraperitoneal pressure in laparoscopy reduces the spread of lymphatic flow.

One patient in this study had histopathologically negative metastatic SLN but positive parametrial nodes; this case was false negative case. Parametrial nodes, specially the ones in the medial part, are essentially difficult to locate with blue dye or the gamma probe because of the proximity of the cervix, where the blue coloration is intense and radioactivity is high. Levenback et al. ${ }^{11}$ and Pijpers et al. ${ }^{12}$ reported false negative results in patients with positive parametrial nodes detected only on final pathologic evaluation of hysterectomy specimen; such involvement does not seem to influence disease free 
survival. ${ }^{13}$ False negative rate in the English medical literatures ranges from $0 \%$ to $50 \%$ with a negative predictive value between $75 \%$ and $100 \%$. To our knowledge, no consensus exists in cervical cancer with regard to our acceptable false negative rate. ${ }^{11}$

Possible adverse effects with detection of SLNs by using dyes are hypersensitivity reactions (more in isotope colloid), blue discoloration of urine, more rarely severe reactions have been reported if dye was mixed with local anesthetic agents. Likewise, falling in the saturation in oximetry probe (pseudo fall down) without clinical consequences. ${ }^{5}$ In this study, most of patients had blue coloration of urine that usually remained not more than 24 hours. No patient had developed anaphylactic reaction.

In this study blue dye was used as $1 \%$ diluted in distilled sterile water and still obtained excellent blue staining of the nodes. The dye was diluted because, it was found that pure dye leads to intense coloration of tissues surrounding cervix and, also to reduce risk of anaphylactic reactions. $^{12}$

Physiology of lymphatic drainage from cervix has been variously described and is not well established. ${ }^{4,13}$ Some authors noted the existence of first level lymph node metastases from early cervical cancer is a random event in that any of the pelvic node sites may be the first stage for metastases. ${ }^{14,15}$ The SLN concept depends on the physiology of lymphatic drainage from the cervix. Giant section studies of surgical specimen obtained at radical hysterectomy showed that lymph nodes are present in the parametrium in most of the patients. ${ }^{14}$

Sakuragi, et al studied the distribution of pelvic lymph node metastases in 208 patients with cervical cancer. ${ }^{15}$ The obturator lymph nodes were most frequently involved and solitary lymph node metastases were found in obturator, internal iliac and parametrial region. This pattern of Iymphatic spread is confirmed by the localization of the sentinel nodes. Acccording to the Dargent, most sentinel nodes are found in pelvic region, near the origin of uterine artery. ${ }^{10}$ This study also showed similar type of presentation of lymph nodes i.e. most common site was obturator, external iliac, parametrial and internal iliac in descending order frequency. In this study no SLNs were found in common iliac and para-aortic region. This could be due to low sample size. So, localization of SLNs in this study was variable but was generally within the area of expected drainage. Parametrial sentinel nodes were identified in $10 \%$ of total SLNs but evaluation of the parametrium was difficult in some cases due to tissue staining. Some patients had multiple sentinel lymph nodes, whether this is a function of delay in identification of the primary drainage tract or a result of multiple independent lymphatic pathways is not known. Unusual site of SLN detection such as the groin have also been reported by two groups. ${ }^{16}$ Burghardt and Pickel advocated complete parametrectomy to the sidewall as integral part of the pelvic lymphadenectomy. ${ }^{17}$ Therefore, is it possible SLN status could reflect parametrial nodal status, considering the parametrial nodes as an adjunctive lymph node group? Therefore, should SLN biopsy have a role in tailoring parametrial resection? There have been many reports discussing the relationship between parametrial and pelvic lymph node metastases, ${ }^{14,18-20}$ some suggesting pelvic lymph node status as a reliable predictor of parametrial involvement. Data from literatures are not uniform and call for further prospective trials aimed at evaluating this hypothesis. At the moment, it is difficult to identify a subset of patients in whom the parametrial tissue can be preserved. Another obvious advantage of sentinel node dissection is to shorten pathologic evaluation and probably render it more accurate since it is limited to one or few nodes.

\section{CONCLUSIONS}

Lymphatic mapping for patients with early cervical cancer undergoing primary surgical therapy appears to be feasible but until the results are available systemic pelvic lymphadenectomy and radical hysterectomy should remain the standard surgical practice for patients with cervical cancer. Based on this preliminary effort, it is believed that further study of Iymphatic mapping technique in patients with cervical cancer is warranted. This small series of study may not be sufficient to make any definitive statement. 


\section{REFERENCES}

1. John DB, Robert LC, Steven GB, et al. Intraoperative lymphatic mapping in cervix cancer patients undergoing radical hysterectomy: A pilot study. Gynecol Oncol. 2000;79:238-43.

2. Magrina JF, Goodrich MA, Lidner TK, Weaver AL, Cornella JL, Podratz KC. Modified radical hysterectomy in the treatment of early squamous cervical cancer. Gynecol Oncol. 1999;72:1836.

3. Gil-Moreno A, Diaz-Feijoo B, Roca I, Puig O, Perez-Benavente MA, Aguilar I, et al. Total laparoscopic radical hysterectomy with intraoperative sentinel node identification in patients with early invasive cervical cancer. Gynecol Oncol. 2005;96(1):18793.

4. Bilchik AJ, Giuliano A, Essner R, Bostick P, Keleman P, Foshag $\mathrm{LJ}$, et al. Universal application of intraoperative lymphatic mapping and sentinel lymphadenectomy in solid neoplasm. Cancer J Sci Am. 1998;4:351-8.

5. Bass SS, Cox CE, Ku NN, Berman C, Reintgen DS. The role of sentinel lymph node biopsy in breast cancer. J Am Coll Surg. 1999;189:183-94.

6. Morton DL, Wen DR, Wong JH, Economou JS, Cagle LA, Storm FK et al. Technical details of intraoperative lymphatic mapping for early stage melanoma. Arch. Surg.1992;127:392-9.

7. Giuliano AE, Kirgan DM, Guenther JM and Morton DL. Lymphatic mapping and sentinel lymphadenectomy for breast cancer. Ann Surg.1994;220:391-401.

8. Echt ML, Finan MA, Hoffman MS, Kline RC, Roberts WS, Fiorica JV. Detection of sentinel lymph nodes with lymphazurin in cervical,uterine and vulvar malignancies. South Med J. 1999;92:204-8.

9. Barranger E, Cortez A, Grahek D, Callard P, Darai E. Laparoscopic sentinel node procedure for cervical cancer: impact of neoadjuvant chemotherapy. Ann Surg. Oncol. 2004;11:175-80.

10. Dargent D, Martin X, Mathevet P. Laparoscopic assessment of the sentinel lymph node in early stage cervical cancer. Gynecol Oncol. 2000;79:411-15.
11. Barranger E, Darai E. Sentinel lymph node biopsy is not accurate in predicting lymph node status for patients with cervical carcinoma. Cancer. 2004;10(8):1919-20.

12. Montgomery LL, Thorne AC, Van Zee KJ, et al. Isosulfan dye reactions during sentinel lymph node mapping for breast cancer. Anesth Analg. 2002;95:385-8.

13. Plentl AA, Friedman EA. Lymphatic system of the female genitalia. In: Friedman EA, editor. Major problems in obstetrics and gynecology vol. 2. Philadelphia: Saunders; 1971:75-115.

14. Benedetti-Panici P, Maneschi F, D'Andrea G, Cutillo G, Rabitti $\mathrm{C}$, Congiu $\mathrm{M}$, et al. Early cervical carcinoma: the natural history of lymph node involvement redefined on the basis of thorough parametrectomy and giant section study. Cancer. 2000; 88:226774.

15. Sakuragi N, Satoh C, Takeda M, Yamamoto R, Fujimotot T, Oikawa $\mathrm{M}$, et al. Incidence and distribution pattern of pelvic and paraaortic lymph node metastasis in patients with stages IB, IIA and IIB cervical carcinoma treated with radical hysterectomy. Cancer. 1999; 85:1574-54.

16. Hauspy J, Verkinderen L, De Pooter C, Dirix LY, Van Dam PA. Sentinel node metastasis in the groin detected by technetium-labeled nannocolloid in a patient with cervical cancer. Gynecol Oncol. 2002;86(3):358-60.

17. Burghardt E, Pickel H. Local spread and lymph node involvement in cervical cancer. Obstet Gynecol. 1976;52:138-43.

18. Covens A, Rosen B, Murphy J, Laframboise S, De Petrillo AD, Lickrish $\mathrm{G}$ et al. How important is removal of the parametrium at surgery for carcinoma of the cervix? Gynecol Oncol. 2002;84(1):145-9.

19. Yen MS, Yuan CC, Wang PH, Ng HT, Twu NF, Juang CM. Parametrial tumor spreading patterns in cervix cancer patients treated by radical hysterectomy. Int J Gynecol Obstet. 2003;80:145-51.

20. Puente R, Guzman S, Israel E, Poblete MT. Do the pelvic lymph nodes predict the parametrial status in cervical cancer stages IB-IIA? Int J Gynecol Cancer. 2004;14:832-40. 